\title{
IDENTIFICATION ET CLASSIFICATION DES TERRES CULTIVABLES ET DES SITES POTENTIELS POUR L'AMENAGEMENT DE RETENUES COLLINAIRES DANS L'EST DU BASSIN MEDITERRANEEN: LE CAS DU HAUT-JBEIL (LIBAN)
}

\author{
Arnaud Caiserman ${ }^{1 *}$, Ghaleb Faour ${ }^{2}$, Dominique Dumas ${ }^{3}$, Karine Bennafla ${ }^{4}$, \\ Georges Chemaly ${ }^{5}$ et Salim Kouroz ${ }^{6}$ \\ ${ }^{1}$ doctorant en Géographie-Aménagement, Université Jean Moulin Lyon 3, France \\ ${ }^{2}$ Directeur du Centre National de Télédétection de Beyrouth (CNRS-L), Mansourieh, \\ Liban \\ ${ }^{3}$ Directeur du Centre de Recherche en Géographie-Aménagement à 1'Université Jean \\ Moulin Lyon 3, France \\ ${ }^{4}$ Directrice du Centre d'études et de documentation économique, juridique et sociale, Le \\ Caire, Egypte \\ ${ }^{5}$ Ingénieur agronome, Plan Vert, Jounieh, Liban \\ ${ }^{6}$ Ingénieur cartographe, Plan Vert, Jounieh, Liban \\ *Auteur correspondant: Arnaud Caiserman \\ arnaudcaiserman@gmail.com
}

(Received July 2018 - Accepted December 2018)

\begin{abstract}
Caiserman, A. Faour, G. Dumas, D. Bennafla, K. Chemaly, G. and Kouroz, S. Identification and classification of cultivable lands and potential sites for hill lakes planning in the East of the Mediterranean basin: the case of the High-Jbeil (Lebanon). Lebanese Science Journal. 19(3): 305 - 329.

In the Mount-Lebanon, the agricultural territory of Aaqoura is significant despite high elevations reaching $2100 \mathrm{~m}$. In the 1960's, apple orchards were subsidized by a state-owned service of planning: the Green Plan, but the increasing urbanization in Lebanon requires nowadays a solid knowledge of the remaining and not yet developed lands for agricultural extension. The challenge of this study is dual: environmental and socio-economic. Two models of potential site identification and location of new cultivable lands and new hill lakes have been developed in this study. These models are based on pedologic, topographic, geologic criteria and simultaneously integrate socio-economic
\end{abstract}

http://dx.doi.org/10.22453/LSJ-019.3.305329 National Council for Scientific Research - Lebanon 2018® lsj.cnrs.edu.lb/vol-19-no-3-2018/ 
surveys with farmers in order to assess the convenience of such new infrastructures and to strengthen its relevance in this region. The results of these models lead to two maps that locate sites with high potential for the implementation of new infrastructure, but its analysis must be completed through survey's results. After field verifications of these maps, $14 \%$ of the area is still available for new agricultural lands and only $4 \%$ for hill lakes on account of steep slopes, permeability of soil, and farmers stressed out economic challenges, such as the lack of profitable markets, which undermines the local agricultural growth. However, farmers are interested in new hill lakes for the purpose of satisfying orchard high water needs. This integrated approach of rural development, appeared as an interesting tool for planning office such as the Green Plan as much as for farmers since they are the first users of these infrastructures.

Key words: irrigated farmings, hill lakes, agricultural lands, rural planning, mount Lebanon

\section{RESUME}

Caiserman, A. Faour, G. Dumas, D. Bennafla, K. Chemaly, G. et Kouroz, S. Identification et classification des terres cultivables et des sites potentiels pour l'aménagement de retenues collinaires dans l'est du bassin méditerranéen : le cas du haut-Jbeil (Liban). Journal Scientifique Libanais. 19(3): 305-329.

Le territoire agricole de Aaqoura dans le Mont-Liban, est important malgré des altitudes élevées allant jusqu'à 2100 m d'altitude. Dans les années 1960, des vergers de pommiers furent subventionnés par le Plan Vert, un service public d'aménagement, mais aujourd'hui, face à l'urbanisation croissante du Liban, la connaissance des terres disponibles pour l'agriculture est primordiale. L'enjeu de cette étude est double: environnemental et socio-économique. Deux modèles d'identification des sites potentiels pour l'aménagement de nouvelles terres cultivables et de retenues collinaires sont proposés. Ces modèles se basent sur de critères pédologiques, topographiques, géologiques tout en intégrant des enquêtes socio-économiques auprès des agriculteurs sur l'utilité de ces nouvelles infrastructures afin de renforcer leur pertinence dans la région. Les résultats aboutissent à deux cartes localisant les sites à fort potentiel pour ces nouveaux aménagements, mais leurs analyses doivent s'accompagner des résultats des enquêtes. Si après validation des cartes sur le terrain, $14 \%$ de la surface du secteur peuvent être équipés en nouvelles terres agricoles et seulement $4 \%$ en retenues collinaires en raison de fortes pentes et de la perméabilité du sol, les agriculteurs ont souligné les difficultés économiques, notamment l'absence de marchés rentables, qui limitent le développement local. En revanche, les agriculteurs montrent un intérêt pour de nouvelles retenues afin de satisfaire pleinement les demandes en eaux des vergers. L'approche 
intégrée de l'aménagement, apparait donc comme un outil intéressant tant pour les bureaux d'étude public comme le Plan vert que pour les agriculteurs eux-mêmes dans la mesure où ces aménagements les concernent directement.

Mots-clés: cultures irriguées, retenues collinaires, terres agricoles, aménagement rural, Mont-Liban

\section{INTRODUCTION}

A l'échelle nationale, le Liban s'inscrit dans une croissance démographique caractéristique du bassin méditerranéen, impliquant une urbanisation des terres au détriment des espaces ruraux et agricoles (Tabarly, 2011). L'agriculture est relativement peu soutenue par le gouvernement qui n'a pas mis en place de réelles politiques agricoles au sortir de la guerre civile dans les années 1990 (Blanc, 2006 ; Chehaita, 2015). Les banques, quant à elles, accordent essentiellement leurs crédits au secteur du service, les agriculteurs n'ayant pas toujours les moyens de les rembourser (World Bank, 2010). Les exploitants de Aaqoura doivent exercer une double profession, afin ade rentabiliser leur activité agricole souvent trop insuffisante (Caiserman, 2015). Aujourd'hui, la crise syrienne pénalise aussi l'agriculture libanaise qui voit ses routes commerciales fermées en direction des pays du Golfe, lesquels constituent des marchés importants pour la vente de ses productions. Les pays voisins, la Jordanie, Territoires palestiniens occupés, l'Egypte et la Turquie s'imposent de plus en plus en concurrents régionaux, et tout particulièrement sur le marché de la pomme. Plus largement à l'ouest, les marchés occidentaux sont difficiles d'accès en raison de leurs fortes exigences en termes de qualité des produits (Frem, 2002), et la moitié des approvisionnements agricoles du sud de la Méditerranée est assurée par les Etats-Unis, le Canada, l'Australie et l'Argentine (Hervieu et al., 2006). Ainsi, au vu de ce contexte économique et politique, on comprend que l'agriculture libanaise se trouve régulièrement en situation déficitaire. En 2009, l'équivalent de 2,29 milliards de dollars de produits agricoles ont été importés alors que seulement 0,42 milliard ont été exporté (Abou Aarrage, 2010). Tout nouvel aménagement agricole gagne donc à être remis dans la perspective économique de l'agriculture libanaise afin d'examiner sa pertinence. L'agriculture étant la principale activité économique du Mont-Liban, la maitrise du foncier agricole est primordiale pour le développement de ce secteur. La chaîne du Mont-Liban est un bon exemple de l'adaptation des cultures en fonction des marchés nationaux et internationaux. Les agriculteurs sont passés progressivement d'une céréaliculture auto-consommatrice à une production de rente avec des plantations de mûriers bombyx, pour les soieries lyonnaises au XIX ${ }^{\text {ème }}$ siècle (Chevalier, 1971), pour enfin, au début des années 60, les remplacer par des pommes en terrasses, plus rentables, cultivées sur des parcelles morcelées de 0,5 à 1 ha (Verdeil et al., 2007 ; Medawar et al., 2008). La surface moyenne des exploitations relativement 
faible reflète une société d'agriculteurs qui n'avaient autrefois que le souci de l'autosuffisance, mais les interventions de l'Etat en matière de développement rural ont fait de cette région un territoire agricole moins marginal et plus productif (Lewis, 1953). Les agriculteurs les plus fortunés ont alors commencé à racheter des terres de propriétaires absents, et ont embauché une main d'œuvre syrienne pour travailler ces vergers dans les années 1960. Afin de rendre l'agriculture plus rentable et plus productive dans le MontLiban, le Plan Vert, service semi-autonome créé en 1963 dépendant du Ministère de l'Agriculture libanais et ayant pour mandat l'aménagement des terres agricoles (Stephan, 2010) a entrepris dans les années 1960 la planification de terres agricoles. Ce programme d'aménagement est aligné sur d'autres exemples internationaux. En effet, de tels bureaux publics d'étude existent aussi dans d'autres pays du bassin méditerranéen comme au Maroc, Tunisie ou encore en Turquie (Faysse, 2015 ; Talineau et al., 1995 ; Mozas et Ghosn, 2013).

Face aux deux grands défis actuels que représentent la croissance démographique et la pression urbaine sur le foncier agricole, le Plan Vert a entrepris de dresser un nouveau modèle multiscalaire capable d'identifier les sites à fort potentiel agricole grâce au Système d'Information Géographique pour l'aménagement de nouvelles terres cultivables et de retenues collinaires, avec des critères pédologiques, topographiques, géologiques mais également socio-économiques. L'intégration de ces différents critères permet d'éviter un problème récurrent: l'approche technique sectorielle (Landais et Deffontaines, 1988) très souvent adoptée dans le bassin méditerranéen par les pouvoirs publics, notamment en Tunisie ou encore en Algérie dans la mesure où les réalités socioéconomiques sont complexes et difficiles à percevoir (Selmi, 2000 ; Habi et al., 2011). Néanmoins, de telles infrastructures comme les retenues collinaires constituent de véritables outils techniques, politiques et sociaux, comme a pu le montrer de nombreuses études notamment en Tunisie, au Maroc, en Algérie et plus généralement dans les pays membres du projet bien connu HydroMed (Heusch, 1986 ; Lacombe, 2007 ; Riaux et al., 2014 ; Ogilvie, 2015). L'implantation de nouvelles retenues collinaires se montre plus efficace lorsqu'elle comprend les facteurs socio-économiques, dont les associations d'irrigants ayant les compétences de gestion et d'entretiens des infrastructures, notamment en Algérie (Zerkaoui et al., 2009).

L'objectif de notre étude est l'identification et la classification de sites potentiels pour l'aménagement de nouvelles terres cultivables et de retenus collinaires à travers la construction de modèles spatialisés et intégrés dans une perspective de développement du territoire agricole, mais également l'évaluation des besoins des agriculteurs en de telles infrastructures, à travers des enquêtes. Dans notre cas d'étude, les modèles cartographiques et les enquêtes ont été réalisé sur la région de Aaqoura, dans le HautJbeil (Figure.1). Cette zone est sélectionnée car il s'agit d'une région fortement agricole, 
composée de nombreuses terrasses agricoles plantées de vergers, et où les retenues collinaires bénéficient de précipitations annuelles importantes, favorables à leur approvisionnement en eau.

Ainsi, la première partie de cette étude présente le secteur de Aaqoura, avec ses caractéristiques physiques et agronomiques afin de mieux cerner les limites et les atouts environnementaux et économiques de la région. De ce premier point découlent les méthodologies employées pour répondre à nos questionnements : les outils cartographiques pour localiser précisément les sites encore disponibles et intéressants à aménager ainsi que des questionnaires et des entretiens semi-directifs avec les agriculteurs pour connaitre leurs attentes et les intégrer à nos résultats qui seront présentés dans un troisième temps. Le dernier point de cette étude consistera en la discussion des résultats et la formulation d'une proposition d'objectifs affinés faite au Plan Vert.

\section{Partie 1: Haut-Jbeil : une zone d'intérêt agricole}

\section{Une topographie accidentée}

La topographie de notre zone d'étude de $128 \mathrm{~km}^{2}$ est relativement accidentée avec de fortes amplitudes altitudinales et des pentes parfois supérieures à 80\% (Photographie. 1 en annexe). Les altitudes passent de $1072 \mathrm{~m}$ à $2081 \mathrm{~m}$ au sommet Jabal Sayidet el Qarn (Figure.1). A l'est se tient le haut plateau karstique constitué par les calcaires du Cénomanien, et délimité par la «falaise blanche» principalement constituée d'éboulements rocheux (Charbel, 2007). Au sud de Mazraat es Siyâd s'écoule le Nahr Ibrahim dans un canyon : ce cours d'eau offre à la région de Aaqoura une source d'eau importante.

Les versants alentours de Aaqoura sont également sensibles aux mouvements de masse, notamment aux coulées de boue qui y sont fréquentes (Chadi, 2013 ; El Hage Hassan et al., 2015). Les fortes intensités des précipitations, l'alternance du gel et du dégel, le pendage des séries géologiques favorisent l'occurrence de coulées sédimentaires, la dernière en date ayant eu lieu en mai 2015 (Photographie. 2 en annexe). Cette dynamique morphogénique est aussi à prendre en compte dans l'implantation d'infrastructures ou d'aménagements de cette région. 


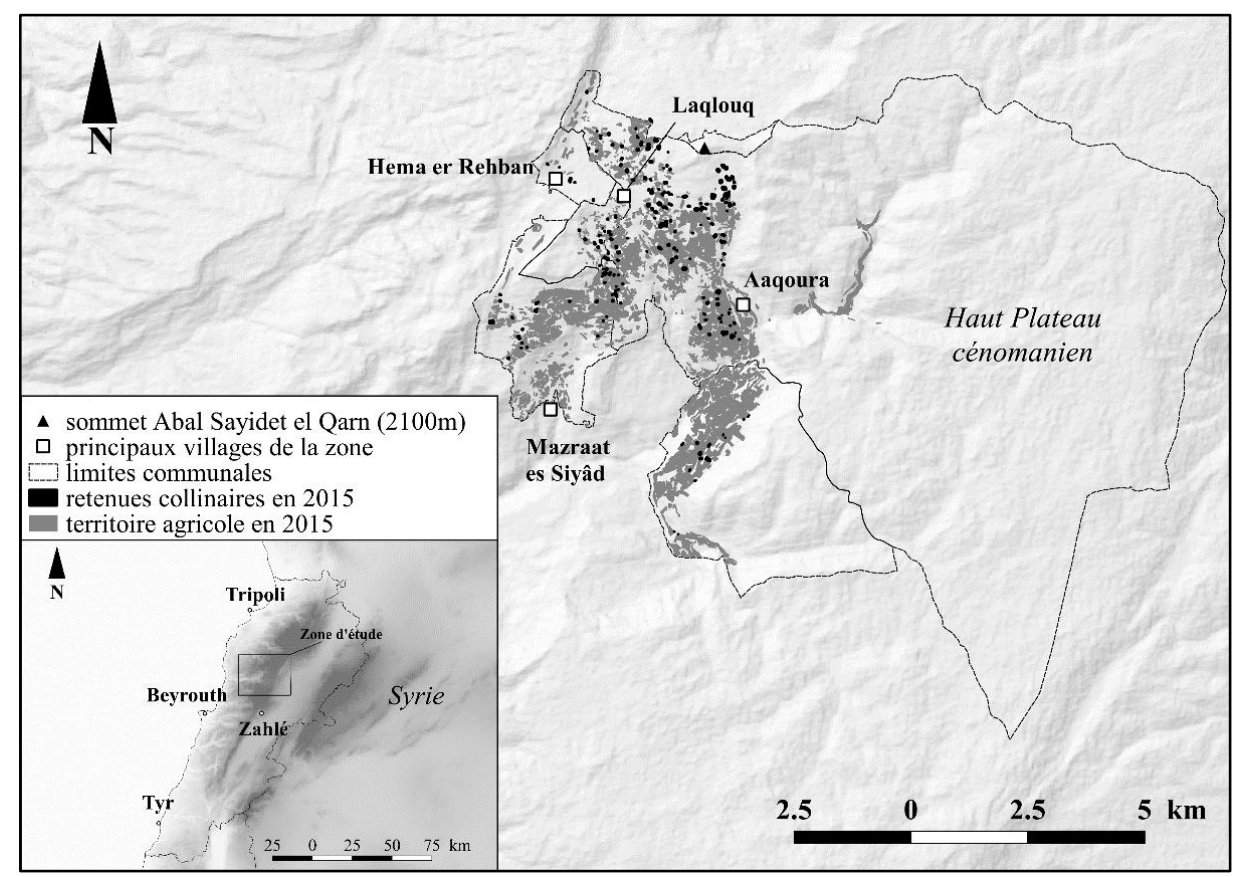

Figure.1 - La région de Aaqoura: terrasses de cultures et retenues collinaires (Sources: National Center of Remote Sensing, 2005. Réalisation; Arnaud Caiserman).

\section{Une région propice à la culture de la pomme}

Malgré cette topographie accidentée et ces fortes pentes, 9\% de la surface de la zone étudiée est occupée par un territoire agricole, principalement réparti sur les pentes inférieures à 40\%, à l'ouest, entre Jabal Sayidet el Qarn au nord et le Nahr Ibrahim au sud (Figure.1). Ces versants sont dominés par des terrasses de cultures en pierre sèche où sont plantées différentes espèces de pommiers : Starking, Golden, Granis ou encore Red Delicious. Avec ces vergers cependant, les besoins en eau sont significatifs. On les estime entre 6000 et $7000 \mathrm{~m}^{3} / \mathrm{ha}$, selon les entretiens avec les agriculteurs, sur toute la saison dont la récolte a lieu entre septembre et octobre. Ces apports en eau pourraient être satisfaits par le climat méditerranéen et montagnard de la région, offrant des précipitations annuelles comprises entre 1500 et $1800 \mathrm{~mm}$ soit 15000 à $18000 \mathrm{~m}^{3} / \mathrm{ha}$ (Charbel, 2007). Cependant, $60 \%$ des précipitations tombent en hiver et au printemps, au moment où les pommiers ont des besoins en eau réduits. Ce régime pluvial rend nécessaire la mise en place de moyens de stockages d'eau, comme les retenues collinaires construites par le Plan Vert dans les années 1960. Au nombre de 203 (Figure 1), ces infrastructures jouent un rôle essentiel dans l'irrigation des pommiers et permettent de combler le manque de précipitations estival. L'utilité de nouvelles terres agricoles et de retenues doit avant tout 
être abordée à travers des enquêtes, afin de comprendre pour quelles raisons les agriculteurs auraient besoin ou non de nouvelles infrastructures.

\section{Partie 2: Les méthodologies employées}

\section{L'enquête auprès des agriculteurs de Aaqoura}

Les agriculteurs, qu'ils soient propriétaires ou locataires, constitueront l'échantillon de cette enquête sous forme de questionnaires et d'entretiens semi-directifs, structurée en trois axes. Au total, 40 enquêtes ont été conduites par les auteurs dans les principaux villages de la zone d'étude, au cours d'une mission de terrain au printemps 2016. Après avoir établi le profil socio-économique de l'enquêté, les besoins en nouvelles terres et retenues collinaires sont évalués en fonction des opportunités économiques pour cette région. Un deuxième axe est prévu pour connaitre les modes de financements des exploitations, dans la mesure où les agriculteurs reçoivent peu d'aides de l'Etat, ce qui peut limiter leurs capacités à entretenir de nouvelles infrastructures. Enfin, la succession des exploitations est abordée à travers plusieurs questions portant sur l'intérêt de la jeunesse pour l'agriculture. Si la jeune génération reprend les exploitations, le développement d'infrastructures ne sera que plus pertinent à long terme.

\section{La classification des critères d'aménagement des terres cultivables et des retenues collinaires}

Le modèle spatialisé d'identification et de classification des sites potentiels repose sur des critères établis par le Plan Vert, représenté dans la figure.2. Les pentes douces inférieures à 20\% selon un Modèle Numérique de Terrain - sont les plus faciles d'accès pour les engins, et moins contraignantes pour les exploitants puisqu'elles ne nécessitent pas de construction de murs de soutènement pour retenir les planches de terrasse. En revanche les pentes supérieures à $40 \%$ sont exclues des modèles en raison des forts coûts de construction dus à un accès plus difficile. Pour les retenues collinaires, les pentes ne doivent pas excéder $30 \%$ en raison de la quantité de matériaux mobilisés trop importantes pour niveler la retenue (Ciron et al., 1984). 


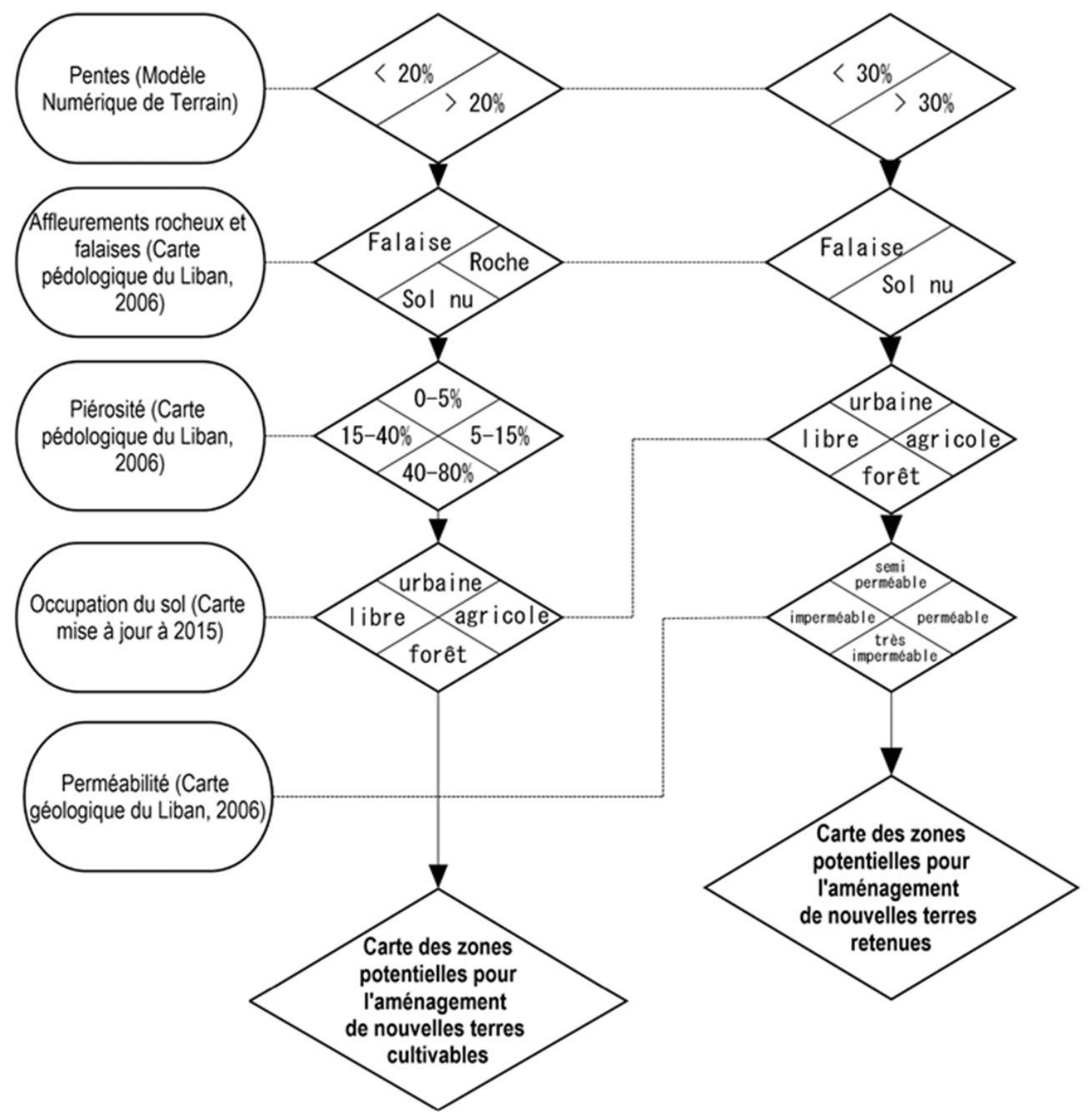

Figure 2. Les critères du Plan Vert pour les modèles cartographiques d'identification et de classification des zones potentielles pour l'aménagement de nouvelles terres et de retenues collinaires.

Aussi, les zones recouvertes de roches nues et les falaises-identifiées à partir de la carte d'occupation du sol de 2005 et en digitalisant les zones ayant une signature spectrale moyenne infrarouge proche des sols rocheux - sont à proscrire car leur destruction représente un coût supplémentaire dans les travaux d'aménagement, tout 
comme les opérations d'épierrage. Ainsi les zones ayant une piérosité supérieure à $80 \%$ selon la carte pédologique du Liban (Darwish, 2006) seront éliminées du modèle. Les classes intermédiaires issues de la carte de 2006 (0-5\%: piérosité faible ; 5-15\%: modérée ; $15-40 \%$ : importante ; 40-80\% : très forte), seront retenues. Les zones à la piérosité faible sont les plus intéressantes à aménager et les zones à très forte piérosité ne sont pas exclues, mais elles seront classées en tant que zones à plus faible potentiel. En revanche, le Plan Vert prévoit un budget d'épierrage et de destruction d'affleurements rocheux pour la construction de retenues collinaires.

Celles-ci ne doivent pas se trouver des couches géologiques perméables où l'installation d'une géomembrane est nécessaire. Notons ici que le Cénomanien est une couche perméable occupant $96 \mathrm{~km}^{2}$ de la surface de la zone (Figure. 3 et tabeau. 1 en annexe), limitant fortement l'aménagement de retenues sans géomembranes. Les couches imperméables (composées de basalte, de tufs, de matériaux volcaniques, de grès ferrugineux et argileux ainsi que de grès quartzeux) seront favorisées et sélectionnées à partir de la carte géologique de la région (Figure.3). Ce critère géologique prévient également les risques de mouvements de masses, en éliminant les zones les zones sensibles cartographiées par Chadi (2013), notamment les formations quaternaires qui correspondent aux passages des coulées de boue.

Enfin les zones sélectionnées par le modèle excluent les territoires urbains, les retenues collinaires déjà existantes, les falaises, les forêts ainsi que la surface agricole déjà en place qu'il a fallu mettre à jour par digitalisation à partir des images satellitaires de 2014 accessibles par Google Earth. Les pentes, les roches nues, la piérosité, la géologie et l'occupation du sol sont donc classées et converties en raster dans Arcmap, afin de ne conserver que les zones susceptibles d'être aménagées.

Les critères d'aménagements sont codés selon leurs importances. Les tableaux.2 et 3 en annexe présentent la matrice et la codification des modèles sur les terres cultivables et les retenues collinaires. La multiplication des critères d'aménagement des terres cultivables donne 7 classes de sites potentiels comme il suit : 0 pour les zones impropres à l'agriculture ; 1 pour les zones à très faible potentiel ; 2 les zones à faible potentiel ; 3 les zones au potentiel moyen ; 4 les zones au potentiel élevé ; 6 les zones à fort potentiel ; 8 les zones à très fort potentiel. Le même procédé de codification est appliqué aux retenues collinaires dont le modèles propose 6 classes différentes. Ces classes vont des zones à potentiel nul aux zones à fort potentiel. Les valeurs de classes 1 et 2 sont regroupées sous la catégorie «faible potentiel» et les classes 4 et 6 sous la catégorie « fort potentiel » pour leurs caractéristiques similaires. 


\section{La validation des modèles cartographiques sur le terrain}

Les zones sélectionnées par les deux modèles ont été validées sur 20 carrés de 250 $\mathrm{m}^{2}$ sur le terrain en mesurant les pentes avec un niveau, l'occupation agricole du sol mise à jour, la présence ou non de roches nues et la piérosité du sol. Ce dernier critère a été validé visuellement sur des carrés de $1 \mathrm{~m}^{2}$ représentatifs de la zone visitée, selon les classes de la carte pédologique de 2006 : 85\% des sites visités sont caractérisés par une piérosité similaire à celle de la carte pédologique.

\section{Partie 3: Les résultats des enquêtes et du modèle cartographique}

\section{Résultats et validation des modèles cartographiques sur les zones potentielles pour l'aménagement de nouvelles terres cultivables et retenues collinaires}

Tout d'abord, 95\% des zones étudiées sur le terrain sont bien dépourvues d'aménagements agricoles, sur des pentes similaires à celles issues du Modèle Numérique de Terrain, et aucune n'est recouverte de roches nues. Les deux modèles sont ainsi considérés comme robustes et capables de sélectionner précisément les zones d'intérêts pour le Plan Vert.

Un extrait de la carte d'identification des zones potentiels pour les nouvelles terres cultivables est donné en figure. 4 en annexe. Il faut tout d'abord relever la catégorie des zones impropres à l'agriculture qui occupent $85 \%$ de la région, notamment en raison de l'occupation agricole du sol déjà bien développée mais aussi des faibles possibilités de nouveaux aménagements compte tenu des nombreuses pentes supérieures à $40 \%$. La forte piérosité du plateau Cénomanien limite aussi la surface disponible pour les terres cultivables. Ensuite se trouve des zones à très faible potentiel du fait de leurs pentes comprises entre 21 et $40 \%$ ainsi qu'une piérosité très forte comprise entre 40 et $80 \%$ d'après la carte pédologique. Ces caractéristiques rendent les zones difficiles à aménager. Les zones à faible potentiel occupent $6 \%$ de la région, principalement dans les dolines de hautes altitudes du plateau Cénomanien où la piérosité y est forte, mais sans roches nues. Au nord de Aaqoura se situent les zones à potentiel moyen, élevé et fort. Chacune de ces catégories occupent moins de $2 \%$ de la région, mais méritent d'être soulignées. Ces trois catégories ont une piérosité du sol inférieure à $40 \%$ et sur des pentes inférieures à $40 \%$. Rappelons ici que les versants de Laqlouq et de Jabal Sayidet el Qarn sont caractérisés par des éboulements rocheux plus complexes à aménager. Cependant la piérosité ne doit pas exclure ces zones sur lesquelles il est possible de retirer les pierres afin d'améliorer le sol. A l'inverse, les zones à très fort potentiel occupent moins de $1 \%$, réparties en 
quelques sites sur toute la région. Les pentes et la piérosité y sont faibles et il n'y a pas de roches nues ni d'infrastructures préexistantes.

Concernant les retenues (extrait de la carte en figure.5 en annexe), 96\% de toute la région étudiée est impropre à l'implantation de nouvelles retenues collinaires selon les critères du Plan Vert, principalement en raison de la présence du plateau Cénomanien sur 75.5\% du secteur de Aaqoura. Du point de vue environnemental, toute cette surface est donc exclue du modèle, à moins d'avoir recours à une géomembrane pour imperméabiliser le fond de la retenue. Dans notre zone d'étude, les agriculteurs désignent aussi les régions de Laqlouq et Hema er Rehban comme intéressantes à aménager. 1\% de cette région a un potentiel faible pour ses pentes inférieures à 30\%, mais dont les couches semi-perméables se situent dans les communes Hema er Rehban ainsi qu'à l'ouest de Aaqoura. Les zones à potentiel modéré occupent quant à elles, $1.5 \%$ de la région avec des pentes comprises entre 10 et $20 \%$ aux couches très imperméables comme l'Albien et le Basalte supérieur sur les versants de Laqlouq. Aussi le secteur de Laqlouq est-il peu équipé en retenues collinaires malgré la présence de terres agricoles, renforçant l'intérêt du Plan Vert et des exploitants pour cette région. Mais c'est la catégorie de zones à fort potentiel qui apparaît comme la plus intéressante à aménager en retenues collinaires puisqu'elles correspond aux couches imperméables voire très imperméables et ce, sur des pentes toujours inférieures à 10\%. Également sur l'Albien et le Basalte Aptien supérieur, ces zones en occupent les pentes les plus faibles sur le relief montagnard de Laqlouq et au sud de Hema er Rehban. Au total, les zones à fort potentiel n'occupent que 132 ha soit $1 \%$ de la région. Il n'en demeure pas moins que cette catégorie constitue la zone prioritaire à investiguer sur le terrain pour établir d'éventuelles retenues collinaires.

\section{Résultats des enquêtes sur l'intérêt des agriculteurs en de nouvelles terres cultivables et en de nouvelles retenues collinaires}

D'après les résultats des enquêtes, la majorité des répondants disent ne pas avoir besoin de nouvelles terres agricoles. $74 \%$ des personnes interrogées possèdent encore des terres non cultivées, et $66 \%$ de ces agriculteurs disent ne pas être prêts à les cultiver. La situation économique de la région de Aaqoura semble défavorable au développement de son secteur agricole en raison des coûts de production élevés, de l'absence de marché et de la surproduction actuelle de pommes. Les marchés sont difficiles à trouver selon les entretiens et les questionnaires dans lesquels $86 \%$ des enquêtés répondent avoir des difficultés à bien vendre leurs productions agricoles car les bénéfices sont passés de 17 à $11 \$$ par caisse de fruit. Cette baisse de profit, de 64\%, s'explique en partie par la perte de marchés internationaux tels que l'Egypte et la Libye, mais aussi les pays du Golfe depuis la fermeture des routes commerciales. Cependant la production de pommes se poursuit, conduisant le Liban à une surproduction de pommes d'environ 220000 T/an en raison 
des difficultés à écouler les stocks, principalement dues à la fermeture des routes commerciales en Syrie (Entretien avec Fathan L, 2016), limitant la pertinence des nouvelles terres cultivables dans la région de Aaqoura. Et Fathan de répondre : «Je ne vois pas l'utilité [...] On a des difficultés à vendre nos pommes donc à quoi ça servirait de nouveaux vergers ? »- ou encore Khaled (2016) - « Semer aujourd'hui (planter de nouveaux pommiers) ça ne sert pas à grand-chose [...] Ce n'est pas le moment, on n'a pas de marché ». Par ailleurs, une des stratégies employées par les propriétaires est le remplacement des pommiers, notamment par la production de vin de table au-dessous de $1500 \mathrm{~m}$ d'altitude, comme a pu le faire Boutros : «On doit vraiment s'éloigner des pommes parce que ça demande beaucoup d'eau et de travail. Ou alors, il faut transformer ces pommes [...] le raisin, plus de cerises... On peut transformer tous ces produits. On peut en faire de l'eau de vie, de l'alcool. ». La transformation de la pomme en jus, compote ou cidre constitue un enjeu économique qui apporterait à ce fruit une valeur ajoutée, et des marchés à ses cultivateurs. Or pour le moment, les pommes sont vendues en vrac à des intermédiaires - damman - sans avoir subi de transformations. La question du financement de l'exploitation agricole semble encore réduire l'utilité de nouvelle terre. Les subventions et les crédits étant rares pour les agriculteurs, ces derniers prélèvent les bénéfices obtenus sur une parcelle, pour les réinvestir sur d'autres, moins productives. «Tout le monde fait comme ça vu qu'on n'a pas d'aide » du gouvernement (Entretien avec Amal, 2016). Les besoins en nouvelles terres agricoles restent donc limités, à moins que le secteur de Aaqoura parvienne à s'intégrer dans des marchés internationaux stables.

Néanmoins, $50 \%$ des personnes interrogées affirment que leurs enfants reprendront l'exploitation familiale, nuançant l'existence d'un fort exode rural, bien qu'incontestablement réel, mais cette donnée pourrait renforcer l'intérêt des nouvelles infrastructures. Par exemple, les enfants de Boutros et Fathan quittent la région pour gagner les villes, mais d'autres enfants d'exploitants sont susceptibles de rester et de succéder à leurs parents, quitte à se constituer en propriétaire urbain, présent seulement les weekends sur leurs terres. Ce type de propriétaire lointain peut constituer une opportunité d'investissements dans les exploitations locales. Si les jeunes générations héritières des terres ne travaillent pas directement la terre, une partie de leurs revenus obtenus dans les centres urbains pourraient être réinvestit dans les terres de Aaqoura. Ce système d'investissement existe déjà depuis la moitié du XX ${ }^{\text {ème }}$ siècle (Lewis, 1953), mais il pourrait se confirmer avec une jeunesse qui reste propriétaire et soucieuse de la rentabilité de l'exploitation malgré son absence.

L'enquête a aussi permis de révéler l'intérêt des enquêtés pour d'éventuelles nouvelles retenues collinaires, notamment à travers leur connaissance des sites potentiels pour les retenues collinaires. Certains d'entre eux évoquent la possibilité d'une grande retenue commune gérée par la municipalité, précisément sur le haut plateau Cénomanien 
que le modèle cartographique éliminait pourtant en raison de sa perméabilité : «On pourrait construire un grand lac pour les exploitants du village, je pense que c'est possible »(Entretien avec Boutros A-Y, 2016). Si certains agriculteurs ont émis des doutes sur les capacités logistiques des municipalités à gérer une telle ressource en eau pour autant d'agriculteurs, d'autres y voient une opportunité de développement de l'irrigation et de la qualité des fruits. La propriété foncière de la région tend à s'individualiser, y compris les retenues, principalement depuis le développement des cultures de rentes comme les pommiers (Germanos-Ghazaly, 1978). Or, plutôt que de multiplier de nouvelles retenues collinaires d'après le simple constat cartographique, il pourrait être intéressant d'en aménager seulement quelques-unes avec une association d'irrigants, comme il peut en exister en Algérie autour de certaines retenues collinaires. La région de Aaqoura n'est pas dotée d'une telle association. Un organisme comme celuici permettrait de gérer et d'entretenir les retenues sur le long terme (Zerkaoui et al., 2009 ; Mozas et al., 2013). De plus, une source d'eau commune permettrait constituer un vecteur de partage des responsabilités et une plus forte coopération entre les agriculteurs puisque le bon entretien d'une retenue en amont des exploitations permet une d'assurer le partage et l'alimentation en eaux pour tous les bénéficiaires ainsi qu'une protection collective contre l'érosion (Cresswell, 1970). Cette « solidarité du travail »(Chevalier, 1968) existe déjà du fait de l'appropriation familiale voire endogamique de la terre. Si les retenues ou les terres sont individualisées, elles sont des propriétés familiales dont les membres travaillent ensemble. Avec cette retenue collinaire imperméabilisée et commune, il s'agirait de reproduire cette appropriation de la terre à la ressource en eau et ce, à l'échelle de la région.

$\mathrm{Au}$ total, les résultats révèlent que seulement $10 \%$ des agriculteurs interrogés déclarent avoir assez d'eau pour irriguer. L'intérêt des exploitants pour ces infrastructures est donc apparent. Le manque de stockage d'eau, bien que les retenues collinaires y soient nombreuses, constitue la première raison de cet intérêt. Khaled précise que le volume d'eau disponible dans la région «n'a jamais suffi pour tout irriguer correctement », au vue de la surface agricole actuelle (Entretien avec Khaled H, 2016). Enfin, des propriétaires agricoles modestes, qui n'ont pas assez de surface pour creuser un réservoir et doivent acheter l'eau à un propriétaire voisin, ont manifesté leur intérêt pour ces équipements et l'investissement dans une retenue collective pourrait être une bonne réponse à leurs situations. 


\section{DISCUSSION}

\section{Simplification du modèle cartographique à l'échelle nationale}

Une localisation fine et détaillée des sites pouvant accueillir ces nouvelles infrastructures concourent à la pérennité des aménagements, parfois construits sur des zones peu adaptées, mais aussi à la maitrise du foncier agricole, dans un contexte d'urbanisation croissante à l'échelle nationale. Les terres cultivables incluent les zones qui peuvent devenir des terres arables pour des cultures temporaires, ou permanentes, comme avec la plantation de vergers (FAO, 2002). Ces terres ne sont donc pas encore aménagées, et demeurent disponibles pour une occupation agricole du sol. Pour irriguer ces nouvelles terres potentielles, des retenues collinaires, capables de stocker l'eau, sont nécessaires. Ce mode de stockage est d'ailleurs répandu dans le bassin méditerranéen, notamment en Grèce, en Tunisie et au Liban (Selmi, 2000 ; Albergel et al., 2004).

Les deux modèles de localisation des sites potentiels ont pour vocation d'être reproduits à l'échelle nationale. Lorsque les terres cultivables ou les retenues collinaires ont une forte utilité, le Plan Vert prévoit de simplifier le modèle cartographique, en supprimant le critère des roches nues qui peuvent être concassées. En revanche, le modèle des terres cultivables gagnerait en précision en ajoutant par exemple la capacité d'infiltration des sols, ou encore le taux de matière organique. La classification des zones disponibles affinerait la sélection en mettant davantage en valeur les terres à fort potentiel agronomique. Concernant le modèle des retenues collinaires, il sera indispensable par la suite d'intégrer des données hydrologiques comme les débits des cours d'eau proches des ouvrages afin d'estimer la faisabilité d'un remplissage des réservoirs sans un impact marqué sur l'eau disponible pour les régions situées à l'aval. La localisation de nouvelles retenues collinaires gagnerait en précision et les capacités de retenue en eau seraient mieux valorisées. Ces études hydrologiques devraient être considérées comme des «préalables systématiques » à l'aménagement de réservoirs (Lacombe, 2007) afin d'assurer leur efficacité du point de vue de l'irrigation.

\section{Intérêts de l'approche intégrée pour le développement rural}

Dans une perspective de reproduction du modèle dans d'autres régions libanaises, une autre préalable semble nécessaire d'après cette étude : l'intégration du travail d'enquête auprès des agriculteurs. Les résultats des enquêtes semblent utiles tant aux décideurs - le Plan Vert - qu'aux bénéficiaires quitte à revoir les objectifs de développement rural. Un projet de développement proposé par les pouvoirs publics qui ne convient pas aux agriculteurs n'est pas une situation inédite dans le bassin Méditerranéen, notamment en Algérie où le «saupoudrage » de retenues collinaires a été 
perçu comme une solution durable pour revitaliser le secteur agricole local (Habi et al., 2013). Au Liban, les discussions avec les agriculteurs semblent souligner les mêmes conclusions mais dans la mesure où cette étude est réalisée avant la construction, elle devrait permettre d'ajuster les plans d'aménagements avec les bénéficiaires. Les modèles de localisation sont précis mais la donnée socio-économique a par exemple permis de révéler l'idée préexistante chez les agriculteurs d'une grande retenue sur le plateau Cénomanien, dont l'imperméabilisation serait rendue possible par les économies faites sur l'abandon de la multiplication d'autres retenues collinaires. Ainsi les résultats du modèle cartographique sont adaptés en fonction des enquêtes. De plus, l'association d'irrigants permettrait d'entretenir cette retenue et son caractère collectif conduiraient les bénéficiaires qui la géreraient à assurer son bon fonctionnement.

Le Plan Vert pourrait donc proposer des subventions à titre collectif, tout en conservant des aides individuelles pour les quelques propriétaires souhaitant construire de nouvelles terres cultivables. Les modèles cartographiques sont suffisamment précis pour identifier des parcelles à fort potentiel et la superposition de ces cartes avec le cadastre identifierait les propriétaires à qui le Plan Vert proposerait des subventions. Dans le même temps, trois autres leviers d'actions seraient intéressants à explorer, non seulement pour la région de Aaqoura, mais aussi pour le Liban et les secteurs agricoles méditerranéens. Le premier consisterait en l'identification de marchés rentables par le gouvernement libanais afin que les agriculteurs puissent exporter leurs productions sur des marchés nationaux et internationaux, d'autant que le Liban fait partie de nombreux accords commerciaux dans la région. L'amélioration de la qualité des fruits et des rendements constitueraient deux autres leviers intéressants pour les agriculteurs. Une production de qualité, avec des rendements plus hauts favoriseraient l'intégration du secteur agricole de Aaqoura et du Liban dans ces marchés internationaux. Rappelons ici que la principale limite des agriculteurs libanais et méditerranéens en général est la difficulté d'accès à des marchés internationaux. La vocation d'aides aux agriculteurs des services publics comme le Plan Vert pourrait remplir cette fonction et renforcer le développement rural. Si de tels marchés sont identifiés, l'amélioration des rendements permettrait de répondre aux nouvelles demandes, sans avoir à étendre de nouvelles terres cultivables dans toute la région (Heusch, 1986), dont le budget public économisé pourrait être réinvestit dans ces deux leviers d'actions.

Si la question, a priori simple, de l'utilité de ces aménagements ne semble pas toujours pertinente lorsque les infrastructures sont déjà construites par l'appareil étatique (Venot et Krishnan, 2011), elle gagne fortement en intérêt dans une étude préalable à l'aménagement, en l'occurrence, de nouvelles terres et de nouvelles retenues collinaires. Ainsi, au-delà de la faisabilité du projet d'aménagement du Plan Vert, cette étude a tenté de constituer une approche intégrée du développement rural, dont les résultats au Liban 
pourraient servir à l'aménagement de structures similaires dans d'autres pays méditerranéens connaissant les mêmes enjeux. L'approche intégrée environnementale et socio-économique et le dialogue avec les agriculteurs permettent de consolider la pertinence du développement rural. La pertinence du développement rural ne peut effectivement pas ignorer les attentes, les capacités économiques et la motivation des agriculteurs locaux à participer à de nouveaux projets d'aménagements (Braden et al., 2009).

\section{CONCLUSION}

Cette étude a montré que l'identification des sites à forts potentiels - tant pour les nouvelles terres que les retenues - est robuste et les nombreux critères du Plan Vert (pente, pierosite, perméabilité, occupation du sol) validés sur le terrain permettent une classification précise des zones d'après les modèles proposés dans cet article. Cette précision est nécessaire pour l'attribution des subventions en fonction des coûts des travaux qui varient selon la pente, la piérosité du sol ou encore la perméabilité des couches géologiques : $14 \%$ de la zone sont encore aménageables en retenues collinaires et $4 \%$, en nouvelles terres agricoles. Cette précision est encore intéressante car elle permet de localiser les zones aux plus forts potentiels pour aménager des terres cultivables, ou des retenues collinaires, respectivement moins de $1 \%$ de la surface de la zone selon les modèles. Ces cartes peuvent ainsi servir à identifier, à l'inverse, les zones à proscrire de l'urbanisation. L'agrégation de ces données topographiques, pédologiques et géologiques devraient permettre l'identification du potentiel agronomique des zones encore nonaménagées, mais il faudrait compléter lesmodèles par des données hydrologiques et pédologiques comme les propriétés chimiques du sol ou son potentiel agronomique. Cette étude a pris le parti d'intégrer les données socio-économiques car la pertinence du développement agricole ne se base pas uniquement sur le plan environnemental : les conditions physiques et socio-économiques doivent être réunies pour qu'une telle politique soit efficace sur le long terme. La discussion avec les acteurs locaux a complété les résultats du modèle cartographique, en localisant d'autres sites intéressants, bien que les sites identifiés par les agriculteurs demandent davantage de travaux. Mais en concentrant ses interventions sur quelques projets d'aménagements, le Plan Vert renforce ainsi ses objectifs de développement rural, évitant de reproduire l'approche sectorielle de l'aménagement, tout en abandonnant pas la dimension environnementale quantitative, sans laquelle aucune étude d'aménagement n'est valable. Le défi, ou l'erreur à ne pas commettre par les bureaux d'étude est bien double : adopter une approche uniquement technique ou une approche seulement socio-économique, la première étant la plus fréquente. Si le développement de nouvelles terres et de retenues semble possible, le développement rural libanais se renforcerait davantage en proposant par exemple des marchés aux propriétaires agricoles, avant d'étendre la surface agricole. 


\section{REFERENCES}

Abou Arrage, J. 2010. Développement et marketing agricole au Liban : le cas des projets de développement de la filière oléicole, Università degli studi di Milano, Milan, $167 \mathrm{pp}$.

Albergel, J. Selmi, S. Balieu, O. 2004. Les petits barrages dans la zone semi-aride méditerranéenne, Mission IRD de Tunis, Tunis el Manzah, 10 pp.

Blanc, P. 2006, Développement régional et cohésion « nationale », Confluences Méditerranée, 56(1): 115-129.

Braden, J.B. Brown, D.G, Dozier, J. Gober, P. Hughes, S.M. Maidment, D.R. Schneider, S.L. Schultz, P.W. Shortle, J.S. Swallow, S.K. Werner, C.M. 2009. Social science in a water observing system, Water Resources Research, 45(11): 1-11.

Caiserman, A. 2015. Le processus d'abandon et de dégradation des terrasses de cultures dans le Haut-Jbeil: le cas de la commune d'Aaqoura, Université Jean Moulin Lyon 3, Lyon, 204 pp.

Chadi, A. 2013. Application of remote sensing and geographical information system for the study of mass movements in Lebanon, Université Pierre et Marie Curie Paris VI, Paris, 255 pp.

Charbel, L. 2007. Études hydrogéomorphologique et hydrokarstique de la région de Laqlouq-Aaqoûra, Université libanaise, Beyrouth, $151 \mathrm{pp}$.

Chehaita, B. Ibrahim, M. 2015. L'agriculture au Liban : la nécessité d'une transition vers l'agriculture durable, National Defense Magazine, 92(1).

Chevalier, D. 1968. Les cadres sociaux de l'économie agraire dans le Proche-Orient au début du XIX ${ }^{\mathrm{e}}$ siècle_: le cas du mont Liban, Revue Historique, 239(1): 87-100.

Chevalier, D. 1971. La société du Mont Liban à l'époque de la Révolution industrielle en Europe, Librairie orientaliste, Paris, 316 pp.

Ciron, P. Sedan, O. 1984. Création de retenues collinaires sur le territoire du parc naturel régional du Luberon : identification des sites potentiels, Bureau de Recherches Géologiques et Minières, Marseille, 55 pp.

Cresswel, R. 1970. Parenté et propriété foncière dans la montagne libanaise, Études rurales, 40(10): 7-79.

Darwish, T. 2006. Soil Map of Lebanon 1: 50 000, Monograph Series 4- National Council for Scientific Research, Beirut, $367 \mathrm{pp}$.

El Hage Hassan, H. Charbel, L. Touchart, L. 2015. Cartographie des conditions de l'érosion hydrique des sols au Mont-Liban : exemple de la région d'El Aaqoura, Physio-géo, 9(1): 141-156.

Fao. 2002. Agriculture mondiale: horizon 2015-2030, Green Ink Ltd, Rome, 96 pp.

Fay, G. 1986. Socio-géographie et projets de développement rural, Tiers-Monde, 27(105): 41-60. 
Faysse, N. 2015. The rationale of the Green Morocco Plan: Missing links between goals andimplementation, Journal of North African Studies, 4(20): 622-634.

Frem, M. 2002. Les modes de coordinations entre agriculteurs et industriels de fruits et légumes au Liban, New Medit, 1(1): 17-26.

Lacombe, G. 2007. Evolution et usages de la ressource en eau dans un bassin versant aménagé semi-aride: le cas du Merguellil en Tunisie Centrale, Université Montpellier II, Montpellier, 306 pp.

Germanos.-Ghazaly, L. 1978. Le paysan, la terre et la femme : organisation sociale d'un village du Mont-Liban, Librairie d'Amérique et d'Orient, Paris, 196 pp.

Habi, M. et Morsli, B. 2011. Contraintes et perspectives des retenues collinaires dans le Nord-Ouest algérien, Sécheresse, 22(1): 49-56.

Hervieu, B. et Abis, S. 2006. Les dynamiques agricoles en Méditerranée, Confluences Méditerranée, 58(3): 169-186.

Heusch, B. 1986. Cinquante ans de banquettes de D.R.S - C.E.S en Afrique du Nord : un bilan, Cahier ORSTOM - Série. Pédologique, 22(2): 153-162.

Landais, E. et Deffontaines, J-P. 1988. Les pratiques des agriculteurs. Point de vue sur un courant nouveau de la recherche agronomique, Etudes rurales, 1(109): 152-158.

Lewis, N. 1953. Lebanon - The mountain and its terraces, Geographical Review, 43(1): 1-14.

Medawar, S. Daoud, R. Rutledge, D. Ouani, N. 2008. Impact économique de la pomiculture pour une agriculture durable en zone de montagne, New Medit, 7(1): 45-49.

Mozas, M. Ghosn, A. 2013. Etats des lieux du secteur de l'eau en Algérie, Ipemed, Paris, $27 \mathrm{pp}$.

National Center of Remote Sensing. 2005. Landcover map of 2005 1: 50 000, CNRS, Beirut.

National Center of Remote Sensing. 2006. Geology Map 1: 250 000, CNRS, Beirut.

Ogilvie, A. 2015. Upscaling water availability and water use assessments in hydro-social systems: the small reservoirs of the Merguellil catchment (Central Tunisia), Université de Montpellier - King's College London, Montpellier-Londres, 329 pp.

Riaux, J. Ogilvie, A. Jenhaoui, Z. 2014. Les retenues collinaires font-elles ressource ? Réflexions à partir de la Tunisie Centrale, Entre abondance et rareté : eau et sociétés dans le monde arabo-méditerranéen et les pays du Sud $-6^{\text {ème }}$ colloque de la T.M.A. for H.S.E.S, Monastir, 25 pp.

Selmi, S. 2000. La gestion collective des lacs collinaires en Tunisie : vide juridique et pouvoir social important, Territoires en mutations, 7(1): 153-164.

Stephan, S. 2010. Ressources terrestres, État de l'environnement et ses tendances au Liban, ECODIT, Beyrouth, 50 pp.

Tabarly, S. 2011. Agricultures sous tension, terres agricoles en extension : des transactions sans frontières, Géoconfluence - ENS Lyon. 
Talineau, J.C. Camus, H. Smaoui,A. 1995. L'environnement lac collinaire : une problématique de recherche et desenjeux pour le développement rural, Cahiers Options Méditerranéennes, 1(9) : 97-107.

Venot, J.P. et Krishnan, J. 2011. Discursive Framing: Debates over Small Reservoirs in the Rural South, Water Alternatives, 4(3): 316-324.

Verdeil, E. Faour, G. Velut, S. 2007. Population et peuplement. Pp.64-90, In: Atlas du Liban : Territoires et Société. Verdeil, E. Faour, G. Velut, E. Presses de l'IFPO, Beyrouth, $205 \mathrm{pp}$.

World Bank. 2010. Lebanon Agriculture Sector Note: Aligning Public Expenditures with Comparative Advantage, World Bank, Washington, D.C, 27 pp.

Zerkaoui, L. Benslimane, M. Hamimed, A. Khaldi, A. 2016. Problématique d'aménagement et conflit d'usage de l'eau en zone de montagne, cas des monts de Beni-Chougrane (nord-ouest algérien), Larhyss Journal, 26(1): 1266-2002. 


\section{ANNEXES}

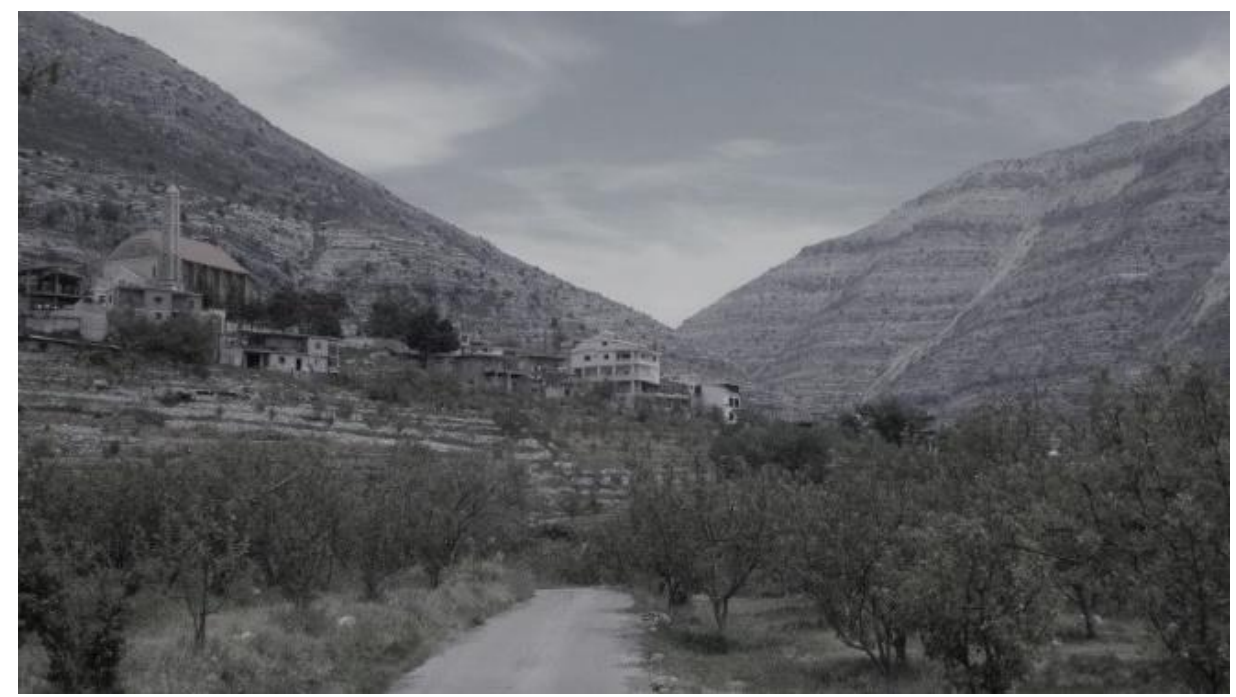

Figure 1. La falaise blanche du Cénomanien en amont des pommiers (Cliché: Arnaud Caiserman, 19 avril 2015).

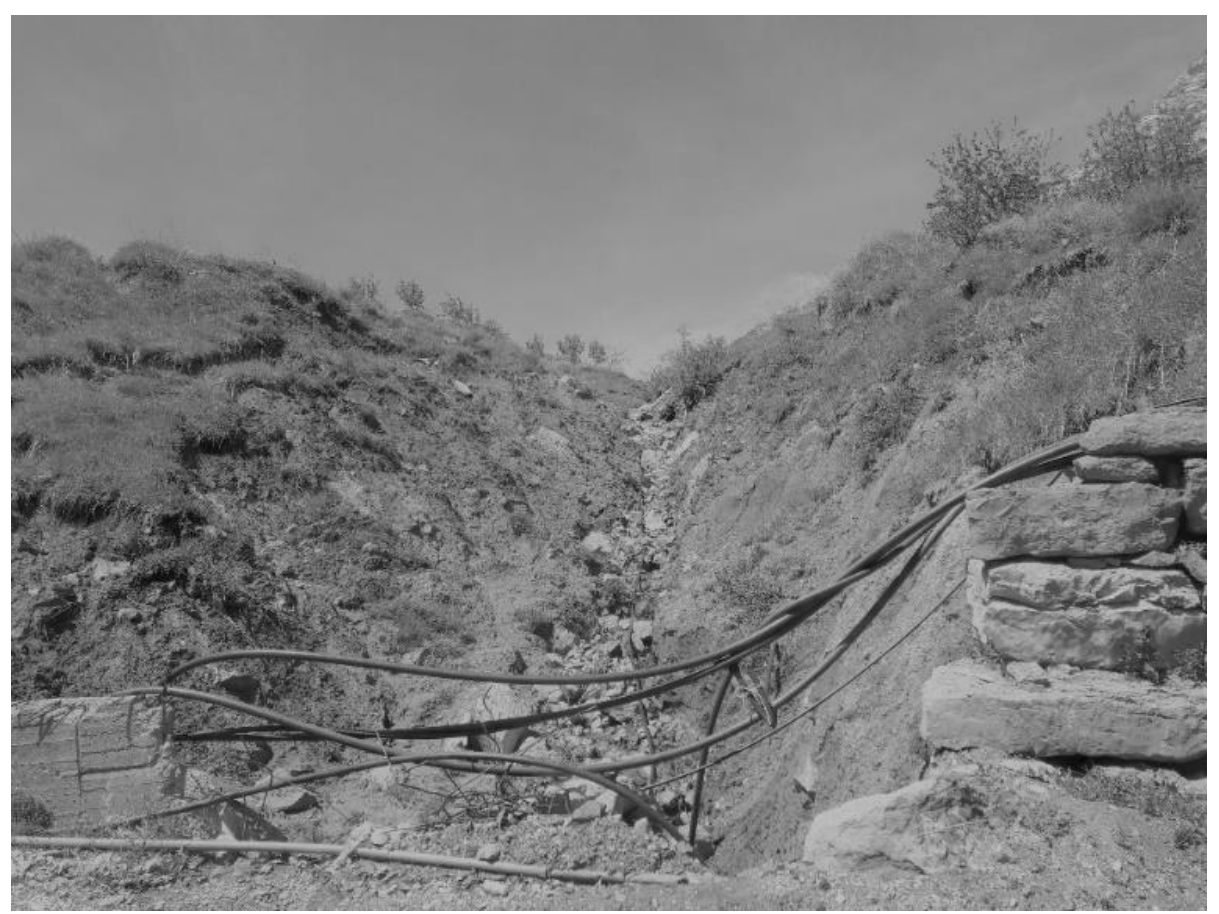

Figure 2. Passage de la coulée de boue de mai 2015 à Aaqoura : témoin de l’instabilité du terrain (Cliché : Arnaud Caiserman, 19 avril 2015). 


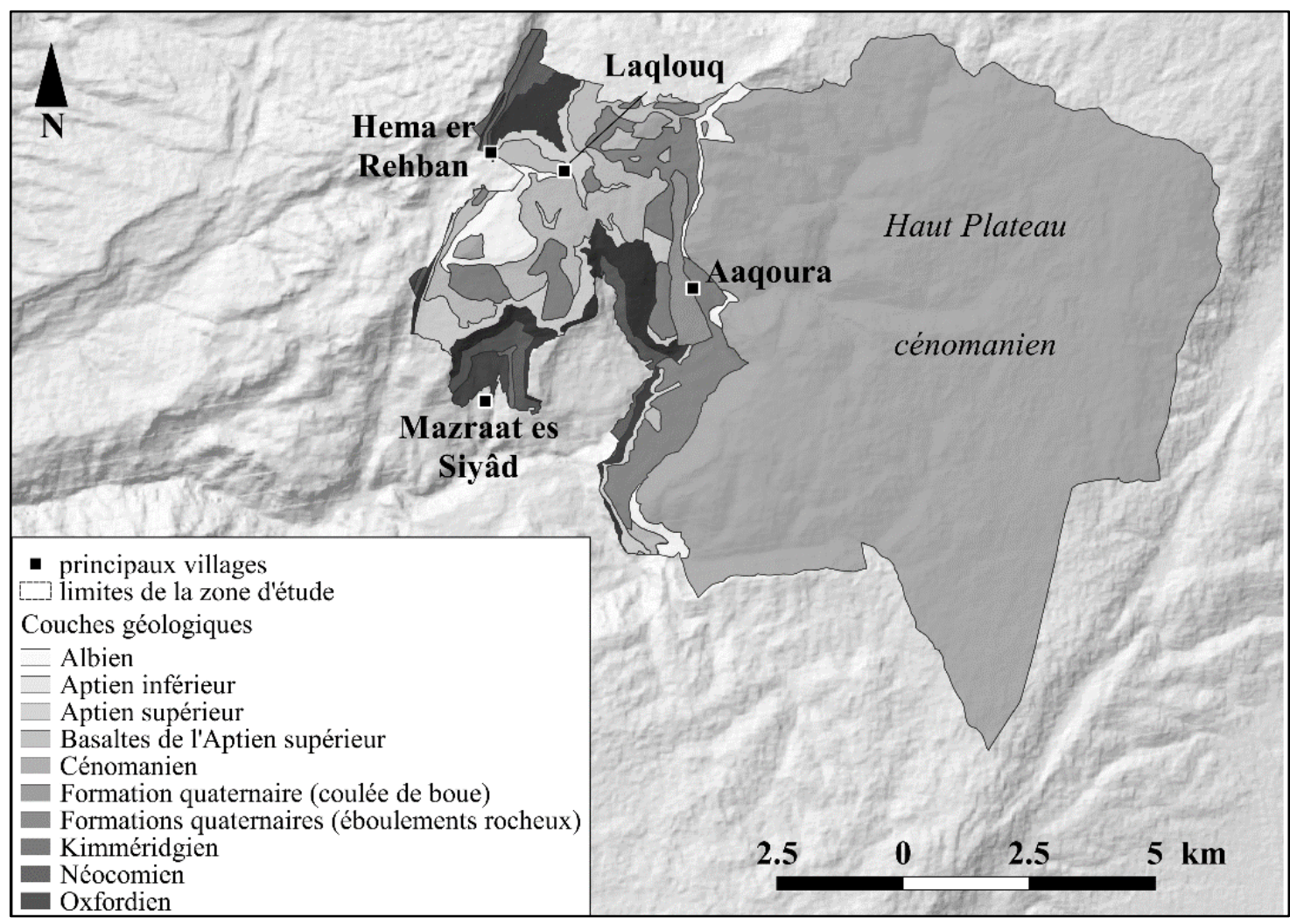

Figure 3. Carte géologique du Haut-Jbeil au 1 :250 000 (Sources: National Center of Remote Sensing, 2006). 
Tableau.1 - Perméabilité des couches présentes dans la zone d'étude (Sources: Charbel, 2007).

\begin{tabular}{|c|c|c|c|c|c|c|}
\hline Période & Époque & Étage & Nature de la couche & \begin{tabular}{|l} 
Surface \\
en $\mathbf{k m}^{2}$
\end{tabular} & $\begin{array}{l}\text { Surface } \\
\text { en \% }\end{array}$ & Perméabilité \\
\hline \multirow{2}{*}{ Quaternaire } & & \begin{tabular}{|c}
$\begin{array}{c}\text { Formation quaternaire } \\
\text { (qe) }\end{array}$ \\
\end{tabular} & éboulis & 8.13 & 6.34 & Perméable \\
\hline & & $\begin{array}{c}\text { Formation quaternaire } \\
(\mathrm{qm})\end{array}$ & coulée de boue & 1.3 & 1.01 & Imperméable \\
\hline \multirow{6}{*}{ Crétacé } & \multirow{2}{*}{$\begin{array}{l}\text { Crétacé } \\
\text { moyen }\end{array}$} & Cénomanien (C4) & $\begin{array}{l}\text { roche carbonatée à } \\
\text { dominante calcaire }\end{array}$ & 96.83 & 75.51 & Perméable \\
\hline & & Albien (C3) & $\begin{array}{c}\text { marnes vertes, } \\
\text { calcaires et dolomies }\end{array}$ & 2.93 & 2.28 & $\begin{array}{c}\text { Très } \\
\text { imperméable }\end{array}$ \\
\hline & \multirow{4}{*}{$\begin{array}{l}\text { Crétacé } \\
\text { inférieur }\end{array}$} & $\begin{array}{c}\text { Basalte de l'aptien } \\
\text { supérieur }(\mathrm{Bc} 2)\end{array}$ & $\begin{array}{c}\text { basaltes, tufs et } \\
\text { matériaux volcaniques }\end{array}$ & 7.18 & 5.60 & $\begin{array}{c}\text { Très } \\
\text { imperméable }\end{array}$ \\
\hline & & $\begin{array}{l}\text { Aptien supérieur } \\
(\mathrm{C} 2 \mathrm{~b})\end{array}$ & $\begin{array}{l}\text { "falaise blanche" } \\
\text { calcaire karstifiée }\end{array}$ & 3.85 & 3.00 & Perméable \\
\hline & & Aptien inférieur (C2a) & $\begin{array}{c}\text { grès quartzeux, } \\
\text { calcaire et argile }\end{array}$ & 4.29 & 3.35 & $\begin{array}{c}\text { Semi- } \\
\text { perméable }\end{array}$ \\
\hline & & Néocomien (C1) & $\begin{array}{l}\text { grès ferrugineux et } \\
\text { argileux }\end{array}$ & 2.36 & 1.84 & Imperméable \\
\hline \multirow{2}{*}{ Jurassique } & \multirow{2}{*}{$\begin{array}{l}\text { Jurassique } \\
\text { supérieur }\end{array}$} & Kimmeridgien (j6) & calcaire & 0.6 & 0.47 & Perméable \\
\hline & & Oxfordien (bJ5) & $\begin{array}{c}\text { basaltes et matériaux } \\
\text { volcanique }\end{array}$ & 0.77 & 0.60 & $\begin{array}{c}\text { Très } \\
\text { imperméable }\end{array}$ \\
\hline
\end{tabular}


Tableau.2 - Matrice du modèle d'identification et de classification des sites potentiels pour les terres cultivables.

\begin{tabular}{|c|c|c|c|c|c|c|}
\hline \multirow{2}{*}{$\begin{array}{c}\text { Roches } \\
\text { nues }\end{array}$} & \multirow{2}{*}{$\begin{array}{c}\text { Occupation } \\
\text { agricole du sol }\end{array}$} & \multicolumn{2}{|c|}{ Piérosité } & \multicolumn{2}{c|}{ Pente } & \multirow{2}{*}{$\begin{array}{c}\text { Valeurs du } \\
\text { modèle* }\end{array}$} \\
\cline { 3 - 6 } & & $\%$ & code & $\%$ & code & \\
\hline 1 & 1 & $0-5 \%$ & 4 & $0-20 \%$ & 2 & 8 \\
\hline 1 & 1 & $0-5 \%$ & 4 & $21-40 \%$ & 1 & 4 \\
\hline 1 & 1 & $0-5 \%$ & 4 & $>40 \%$ & 0 & 0 \\
\hline 1 & 1 & $6-15 \%$ & 3 & $0-20 \%$ & 2 & 6 \\
\hline 1 & 1 & $6-15 \%$ & 3 & $21-40 \%$ & 1 & 3 \\
\hline 1 & 1 & $6-15 \%$ & 3 & $>40 \%$ & 0 & 0 \\
\hline 1 & 1 & $16-40 \%$ & 2 & $0-20 \%$ & 2 & 4 \\
\hline 1 & 1 & $16-40 \%$ & 2 & $21-40 \%$ & 1 & 2 \\
\hline 1 & 1 & $16-40 \%$ & 2 & $>40 \%$ & 0 & 0 \\
\hline 1 & 1 & $41-80 \%$ & 1 & $0-20 \%$ & 2 & 2 \\
\hline 1 & 1 & $41-80 \%$ & 1 & $21-40 \%$ & 1 & 1 \\
\hline 1 & 1 & $41-80 \%$ & 1 & $>40 \%$ & 0 & 0 \\
\hline 1 & 1 & $81-100 \%$ & 0 & $0-20 \%$ & 2 & 0 \\
\hline 1 & 1 & $81-100 \%$ & 0 & $21-40 \%$ & 1 & 0 \\
\hline 1 & 1 & $81-100 \%$ & 0 & $>40 \%$ & 0 & 0 \\
\hline 0 & 1 & 1 & & 0 & & 0 \\
\hline
\end{tabular}

$* 0=$ zone impropre à l'agriculture $1=$ très faible potentiel $; 2=$ faible potentiel $; 3=$ potentiel moyen ; $4=$ potentiel élevé $; 6=$ fort potentiel $; 8=$ très fort potentiel 
Tableau.3 - Matrice du modèle d'identification et de classification des sites potentiels pour les retenues collinaires.

\begin{tabular}{|c|c|c|c|c|c|c|c|}
\hline \multicolumn{6}{|c|}{ Facteurs } & \multirow{2}{*}{\multicolumn{2}{|c|}{ leurs du modèle* }} \\
\hline Pente & \multicolumn{2}{|c|}{$\begin{array}{l}\text { Perméabilité } \\
\text { géologique }\end{array}$} & $\begin{array}{c}\text { occupation } \\
\text { du sol (2015) }\end{array}$ & \multicolumn{2}{|c|}{$\begin{array}{c}\text { mouvement } \\
\text { de masse }\end{array}$} & & \\
\hline $\begin{array}{c}\text { classe en } \\
\%\end{array}$ & code & & sse & $\begin{array}{c}\text { valeur } \\
\text { par } \\
\text { pixel }\end{array}$ & $\begin{array}{c}\text { code } \\
\text { (zone } \\
\text { "libres"' }\end{array}$ & \begin{tabular}{|c} 
code \\
(zone \\
sans \\
M.M) \\
\end{tabular} & \\
\hline $0-10 \%$ & 2 & \multirow{3}{*}{\multicolumn{2}{|c|}{ perméable }} & 0 & \multirow{12}{*}{1} & \multirow{12}{*}{1} & 0 \\
\hline $11-30 \%$ & 1 & & & 0 & & & 0 \\
\hline$>31 \%$ & 0 & & & 0 & & & 0 \\
\hline $0-10 \%$ & 2 & \multirow{3}{*}{\multicolumn{2}{|c|}{ semi-perméable }} & 1 & & & 2 \\
\hline $11-30 \%$ & 1 & & & 1 & & & 1 \\
\hline$>31 \%$ & 0 & & & 1 & & & 0 \\
\hline $0-10 \%$ & 2 & \multirow{3}{*}{\multicolumn{2}{|c|}{ imperméable }} & 2 & & & 4 \\
\hline $11-30 \%$ & 1 & & & 2 & & & 2 \\
\hline$>31 \%$ & 0 & & & 2 & & & 0 \\
\hline $0-10 \%$ & 2 & \multirow{3}{*}{\multicolumn{2}{|c|}{ très imperméable }} & 3 & & & 6 \\
\hline $11-30 \%$ & 1 & & & 3 & & & 3 \\
\hline$>31 \%$ & 0 & & & 3 & & & 0 \\
\hline
\end{tabular}

* $0=$ impropre à l'implantation de retenues $1=$ potentiel très faible $; 2=$ potentiel faible $; 3=$ potentiel modéré $; 4=$ fort potentiel $; 6=$ très fort potentiel 


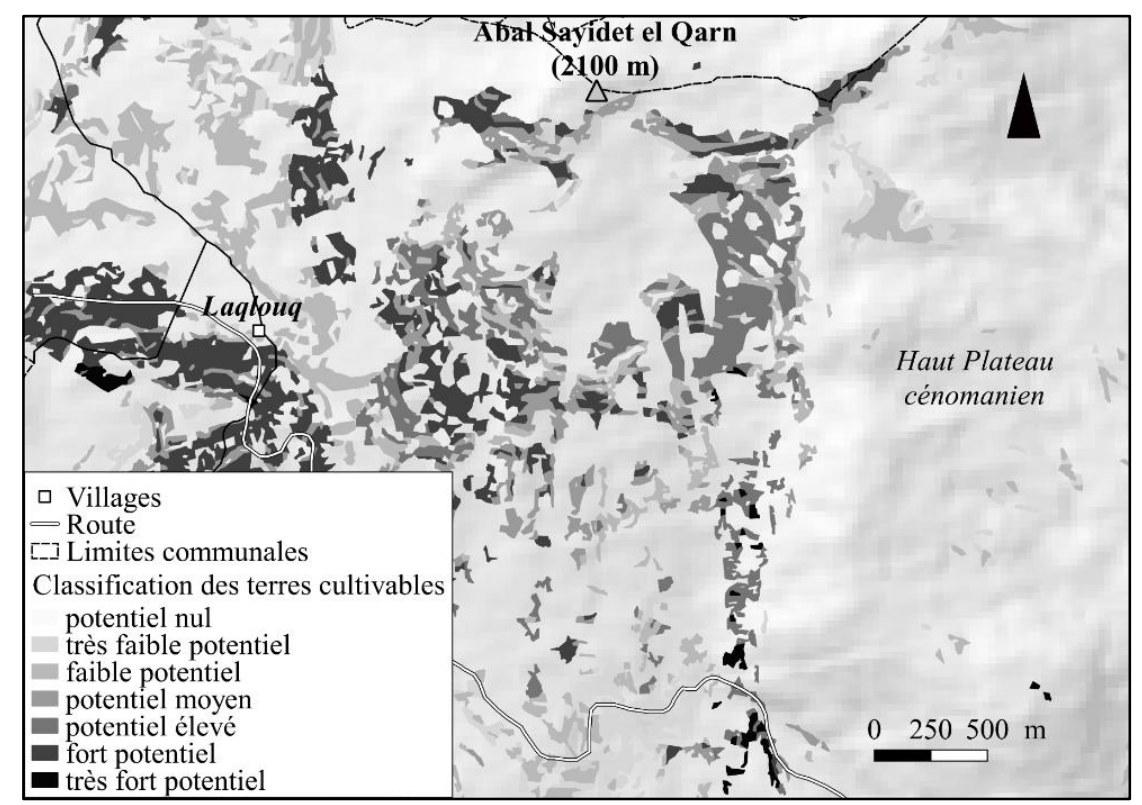

Figure 4. Extrait de la carte d'identification et de localisation des terres cultivables dans la région de Aaqoura (Sources: National Center of Remote Sensing, 2005.

Réalisation: A. Caiserman).

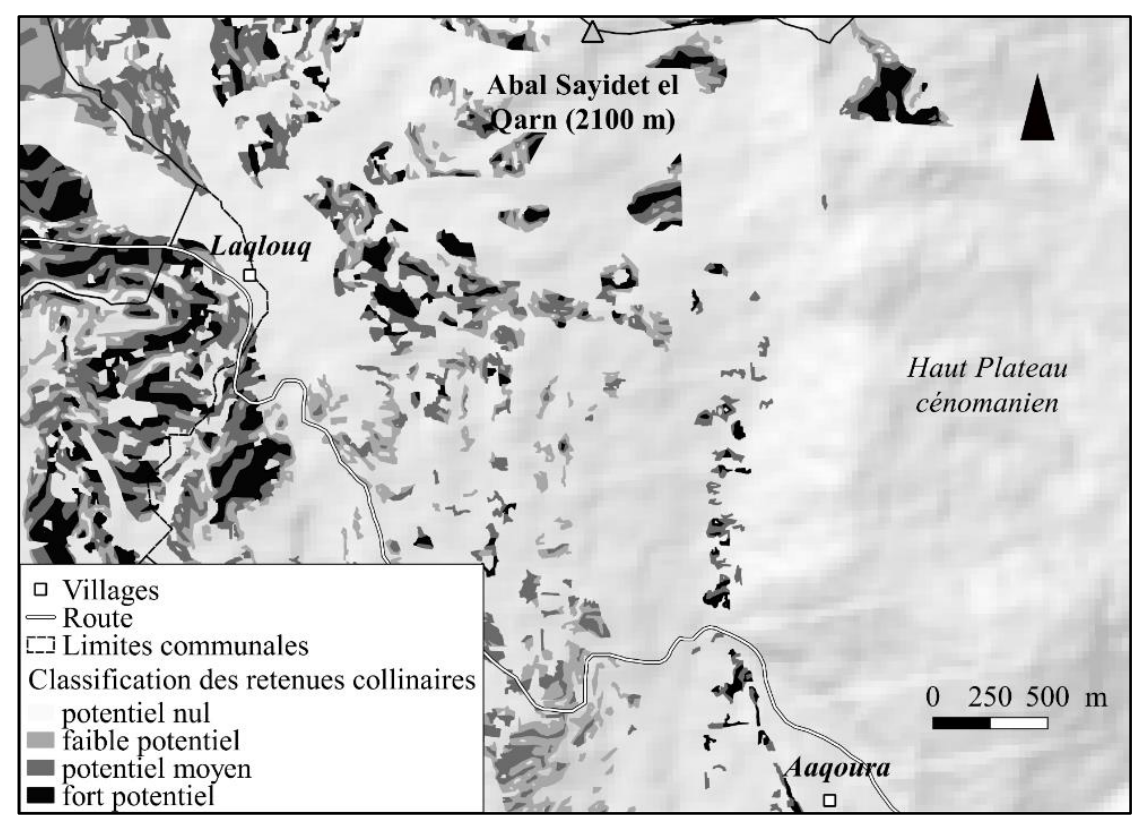

Figure 5. Extrait de la carte d'identification et de localisation des sites potentiels pour l'aménagement de retenues collinaires dans la région de Aaqoura (Sources: National Center of Remote Sensing, 2005-2006. Réalisation : A. Caiserman). 